\title{
Expression of Aquaporin-4 in Fast-Twitch Fibers of Mammalian Skeletal Muscle
}

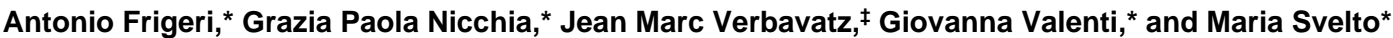 \\ *Dipartimento di Fisiologia Generale ed Ambientale, Università degli Studi di Bari, 70126 Bari, Italy; and ${ }^{\ddagger}$ Service de Biologie Cellulaire, \\ Centre Etudes Nucleaires de Saclay, 91191 Gif sur Yvette, France
}

\begin{abstract}
In this study we analyzed the expression of aquaporin-4 (AQP4) in mammalian skeletal muscle. Immunohistochemical experiments revealed that affinity-purified AQP4 antibodies stained selectively the sarcolemma of fast-twitch fibers. By immunogold electron microscopy, little or no intracellular labeling was detected. Western blot analysis showed the presence of two immunopositive bands with apparent molecular masses of 30 and $32 \mathrm{kD}$ specifically present in membrane fraction of a fast-twitch rat skeletal muscle (extensor digitorum longus, EDL) and not revealed in a slow-twitch muscle (soleus). PCR Southern blot experiments resulted in a selective amplification in EDL of a 960-bp cDNA fragment encoding for the full-length rat form of AQP4. Functional experiments carried out on isolated skeletal muscle bundle fibers demonstrated that the osmotic response is faster in EDL than in soleus fibers isolated from the same rat. These results provide for the first time evidence for the expression of an aquaporin in skeletal muscle correlated to a specific fiber-type metabolism. Furthermore, we have analyzed AQP4 expression in skeletal muscle of mdx mice in which a decreased density of orthogonal arrays of particles, a typical morphological feature of AQP4, has been reported. Immunofluorescence experiments showed a marked reduction of AQP4 expression suggesting a critical role in the membrane alteration of Duchenne muscular dystrophy. (J. Clin. Invest. 1998. 102:695-703.) Key terms: water transport • aquaporin-4 - fast-twitch fibers • orthogonal arrays • Duchenne muscular dystrophy
\end{abstract}

\section{Introduction}

The aquaporin family of membrane water transport proteins confers high membrane water permeability to certain tissues in animals, plants, and microorganisms $(1,2)$. The mercurial insensitive water channel aquaporin-4 (AQP4/MIWC) ${ }^{1}$ initially cloned from rat lung (3) is a nonglycosylated protein with six putative transmembrane domains lacking cysteines at the

Antonio Frigeri and Grazia Paola Nicchia contributed equally to this study.

Address correspondence to Maria Svelto, Ph.D., Dipartimento di Fisiologia Generale ed Ambientale, Università degli Studi di Bari, via Amendola 165/A, 70126 Bari, Italy. Phone: 39-80-5442928; FAX: 3980-5443388; E-mail: m.svelto@biologia.uniba.it

Received for publication 17 December 1997 and accepted in revised form 10 June 1998.

J. Clin. Invest.

(C) The American Society for Clinical Investigation, Inc. 0021-9738/98/08/0695/09 \$2.00

Volume 102, Number 4, August 1998, 695-703

http://www.jci.org known mercury-sensitive sites. Two initiation sites have been identified encoding polypeptides of 301 and 323 amino acids (4) which when expressed in Xenopus oocytes conferred a 20fold increase in $\mathrm{HgCl}_{2}$-insensitive osmotic water permeability. AQP4 is predominantly expressed in cerebellum and in periventricular tissues in brain (4) where it has been proposed to play an important role in a number of physiologic and pathologic processes such as normal pressure hydrocephalus, pseudotumor cerebri, and postischemic brain edema.

AQP4 has also been localized in other tissues including the basolateral plasma membrane of renal collecting duct principal cells, airway epithelium, gastric parietal cells, and skeletal muscle plasmalemma $(5,6)$. These sites correspond to membranes where orthogonally aggregated 6-7-nm particles clustered with a square lattice spacing of $7 \mathrm{~nm}$ (orthogonal arrays of particles, OAPs) have been observed by freeze-fracture electron microscopy. This lead to the hypothesis that AQP4 could be the OAP protein (6). Direct evidence for this hypothesis came from Chinese hamster ovary cells stably transfected with the coding sequence of rat AQP4. These cells showed distinct OAPs on the plasma membrane P-face not observed in control cells and provided direct evidence that AQP4 can spontaneously assemble in regular arrays (7). Interestingly, modification of the membrane content of these OAPs has been reported for various pathological states including brain ischemia $(8,9)$, alumina-induced epilepsy $(10)$, and muscular dystrophy (11).

Recently, AQP4 expression has been reported in skeletal muscle by RNA protection assay and immunoperoxidase staining (6). No expression was found in cardiac or in smooth muscle (6).

In skeletal muscle a redistribution of water and ions between body fluid compartments during exercise causes ionic alterations in muscle and blood as water moves from the plasma $(12,13)$ into both the interstitial and intracellular fluid compartments of contracting skeletal muscle. The main driving force for the net flux of water into contracting muscle is the increase in intracellular osmolarity due mainly to the rapid hydrolysis of phosphocreatine as well as to the increase in lactate accumulation resulting from the increased rate of glycolysis. Lactate accumulation is the greatest single contributor to the osmotic changes. In fact, a high correlation has been found between the increase in muscle lactate and water content (14). However, the molecular mechanism of water permeation across the sarcolemma of skeletal muscle is unknown.

The purpose of this study was to investigate the expression

1. Abbreviations used in this paper: AQP4, aquaporin-4; CMF-PBS, $\mathrm{Ca}^{2+}, \mathrm{Mg}^{2+}$-free PBS; CMTMR, chloro-methyl-benzoyl-amino-tetramethyl-rhodamine; COX, cytochrome $c$ oxidase; DMD, Duchenne muscular dystrophy; EDL, extensor digitorum longus; FG, fast-twitch glycolytic; FOG, fast-twitch oxidative glycolytic; OAPs, orthogonal arrays of particles; SO, slow-twitch oxidative; TIR, total internal reflection. 
of AQP4 protein in mammalian skeletal muscle. Functional measurements were performed in muscles with different fibertype composition in order to characterize the osmotic properties of fast and slow muscles. Further, affinity-purified polyclonal antibodies localized AQP4 exclusively at the sarcolemma of fast-twitch fibers in which water transport measurements indicate that a temperature-independent water movement occurs, and where OAPs are mainly located. Southern PCR experiments indicate that strong differences at messenger level in AQP4 expression exist between the two fiber types. A drastic reduction in the AQP4 expression was finally found in $\mathrm{mdx}$ mouse skeletal muscle, a dystrophin-deficient animal model for X-linked Duchenne muscular dystrophy (DMD) in which a decreased density of OAPs has been reported (11).

\section{Methods}

Animals and biopsies. Pathogen-free male Wistar rats weighing between 250 and $350 \mathrm{~g}$ were obtained from Stefano Morini S.A.S. (Reggio Emilia, Italy). The animals were maintained in a pathogen-free state and were fed ad libitum with autoclaved commercial rodent diet (Charles River, Lecco, Italy) and had free access to drinking water.

Female mdx (C57BL10ScSn mdx) and control mice were supplied from the animal facility of the Policlinico Gemelli (courtesy of Dr. R. Filippetti, Catholic University of Rome, Rome, Italy) and studied at 5 mo of age. Mice were killed by cervical dislocation and the bilateral tibialis anterior muscles were excised.

Human skeletal muscle was obtained from biopsies after surgery (Clinica S. Maria, Bari, Italy).

Preparation of muscle membrane fractions. Rats were killed by decapitation and total skeletal muscle, extensor digitorum longus (EDL), soleus muscles, heart, and brain were isolated and rapidly placed into cold $\left(4^{\circ} \mathrm{C}\right) 5 \mathrm{mM}$ imidazole, $0.3 \mathrm{M}$ sucrose ( $\left.\mathrm{pH} 7.4\right)$, containing protease inhibitors $(1 \mu \mathrm{g} / \mathrm{ml}$ leupeptin, $1 \mu \mathrm{g} / \mathrm{ml}$ pepstatin, and $1 \mathrm{mM}$ PMSF). Skeletal muscles, heart, and brain were minced with scissors, washed with imidazole-sucrose buffer, and transferred to 10 vol of fresh imidazole-sucrose and then homogenized in a Polytron homogenizer for $30 \mathrm{~s}$ at half-maximal speed and centrifuged at $1,000 \mathrm{~g}$ for $10 \mathrm{~min}$. The supernatant was saved and filtered throughout a nylon mesh. Sequential centrifugations of the filtrate were carried out at $4,000 \mathrm{~g}$ for $10 \mathrm{~min}, 17,000 \mathrm{~g}$ for $30 \mathrm{~min}$, and $100,000 \mathrm{~g}$ for $1 \mathrm{~h}$. The pellets from these centrifugations were resuspended in imidazolesucrose and protein content was measured using a modified Lowry procedure (15).

SDS-PAGE and Western blot analysis. Polyclonal antibodies against the COOH terminus of AQP4 (amino acids 287-301, EKGKDSSGEVLSSV) were raised in rabbit as described previously (5).

Rat membrane fractions were solubilized in Laemmli sample buffer, heated at $60^{\circ} \mathrm{C}$ for $10 \mathrm{~min}$, and resolved on a $13 \%$ polyacrylamide gel. Separated proteins were stained with Coomassie brilliant blue or were electrotransferred (for $1 \mathrm{~h}$ at $0.8 \mathrm{~mA} / \mathrm{cm}^{2}$ ) to a PVDF membrane for immunoblot analysis. After transfer, the membrane containing blotted proteins was blocked with Blotto containing 5\% nonfat dry milk for $1 \mathrm{~h}$ and incubated for $2 \mathrm{~h}$ with AQP4 immune serum or with AQP4 affinity-purified antibodies at $0.3-0.5 \mu \mathrm{g} / \mathrm{ml}$. After three washings with blocking buffer, the membrane was incubated for $1 \mathrm{~h}$ with peroxidase-conjugated goat anti-rabbit antibody (Sigma Chemical Co., St. Louis, MO) diluted 1:5,000 in blocking buffer, washed again, and revealed for peroxidase activity by enhanced chemiluminescence (ECL ${ }^{+}$; Amersham, Arlington Heights, IL).

Sequence analysis. Sequence analysis was performed at the Harvard Microchemistry Facility (Cambridge, MA). In brief, after SDSPAGE, the gel was stained with Coomassie blue. Gel pieces containing the $25-\mathrm{kD}$ protein were incubated overnight with trypsin and extracted peptides were subjected to reverse-phase HPLC. Molecular masses were obtained using matrix-assisted laser desorption time- of-flight mass spectrometry. Selected peaks were processed for sequence analysis using the Edman procedure.

Immunomicroscopy. For immunofluorescence, EDL and soleus muscles from rat, human rectum abdominal muscle, and EDL from $\mathrm{mdx}$ mice were cut into small pieces and flash frozen in isopentane cooled in liquid nitrogen. 5-8- $\mu \mathrm{m}$ cryostat sections, mounted on silaned microscope slides, were kept in PBS for $10 \mathrm{~min}$ and preincubated with $1 \%$ BSA in PBS for 15 min as blocking step. Then, sections were incubated with affinity-purified AQP4 antibodies (0.3-0.5 $\mu \mathrm{g} / \mathrm{ml}$ ) for $1 \mathrm{~h}$ at room temperature. Control experiments were performed using purified antibodies preabsorbed with an excess of synthetic peptide as previously described (5). The slides were washed in PBS supplemented with $2.7 \% \mathrm{NaCl}$ (high salt PBS) and then in regular PBS. The sections were then incubated for $1 \mathrm{~h}$ with CY3-coupled goat anti-rabbit antibody (diluted 1:200; Sigma Chemical Co.) followed by washes as above. In some experiments after incubation with the secondary antibody, the slides were treated for cytochrome $c$ oxidase activity (COX) (16). Sections were finally mounted in PBS/ glycerol (1:1) containing $1 \% n$-propylgallate, $\mathrm{pH} 8$. Sections were examined with Nikon Microphot photomicroscope equipped for epifluorescence and photographed using Kodak TMAX 400 film.

For immunogold electron microscopy, muscle samples were fixed in PBS containing paraformaldehyde $4 \%$ for $3 \mathrm{~h}$ and washed several times in PBS. Small pieces of muscle tissue were imbedded in unicryl (British BioCell International, Cardiff, United Kingdom). 70-nm sections were cut on a Reichert-S ultramicrotome (Leica, Wetzlar, Germany) and collected on formar-coated electron microscope grids. Sections were preincubated in T buffer containing $0.1 \%$ BSA, $0.05 \%$ Tween 20 in $100 \mathrm{mM}$ Tris- $\mathrm{HCl}, \mathrm{pH}$ 7.5, for $30 \mathrm{~min}$, then incubated in primary antibody (1:5 dilution of affinity-purified anti-AQP4 antibody) for $2 \mathrm{~h}$, washed $6 \times 5 \mathrm{~min}$ in $\mathrm{T}$ buffer and incubated in a 1:20 dilution of $10-\mathrm{nm}$ gold-conjugated goat anti-rabbit secondary antibody (Amersham) in $\mathrm{T}$ buffer for $45 \mathrm{~min}$ and washed $6 \times 5 \mathrm{~min}$ in $\mathrm{T}$ buffer. Finally, sections were stained with $5 \%$ uranyl acetate for 3 min, then with lead-citrate for $1 \mathrm{~min}$, dried, and observed with an electron microscope (EM 400; Philips, Eindhoven, The Netherlands).

$R N A$ preparation and Southern PCR. Total RNA was prepared from rat brain, EDL, and soleus using the TRIzol Reagent (GIBCO BRL, Gaithersburg, MD), following the manufacturer's instructions. For PCR Southern blot analysis, cDNAs were reverse transcribed from $10 \mu \mathrm{g}$ of rat brain, EDL, and soleus total RNA using oligo-dT primers and Superscript II reverse transcriptase (GIBCO BRL). After RNAase $\mathrm{H}$ treatment, the cDNAs were PCR amplified $\left(94^{\circ} \mathrm{C}, 1\right.$ min; $55^{\circ} \mathrm{C}, 1 \mathrm{~min} ; 72^{\circ} \mathrm{C}, 2 \mathrm{~min} ; 30$ cycles) using specific primers for AQP4 rat sequence (4): 5'-ATGAGTGACGGAGCTGCAGC-3' corresponding to bp +1 to +20 (sense) and 5'-TACAGAAGATAATACCTCTC-3' corresponding to bp +950 to +969 (antisense). Histone 3.3a was used as internal standard for PCR (forward primer: 5'-ACTGGTGGTAAAGCACCCAG-3', reverse primer: 5' TGTCCCCTATTTTCCACTCG-3'). Controls without the addition of reverse transcriptase were run in parallel to rule out genomic amplification and a water blank was included in each assay to detect any contamination. The PCR products, separated on $1 \%$ agarose gel, were visualized by staining with ethidium bromide and photographed. The specificity of the PCR-amplified products was further verified by Southern hybridization. PCR products were transferred onto a nylon membrane and UV cross-linked. The membrane was probed at high stringency in $6 \times$ SSC, $5 \times$ Denhardt's solution, $1 \%$ SDS, $10 \mathrm{mM}$ Tris- $\mathrm{HCl}, \mathrm{pH} 7.4$, at $65^{\circ} \mathrm{C}$ for $16 \mathrm{~h}$ with a ${ }^{32} \mathrm{P}$-labeled cDNA corresponding to the rat AQP4 coding region. The PCR Southern blot membrane was autoradiographed with Hyperfilm-MP (Amersham) for $30 \mathrm{~min}$ at room temperature.

Isolation of fibers and total internal reflection (TIR) experiments. Functional studies were done on rat EDL and soleus isolated fibers. Muscles from killed rats were rapidly removed and placed into cold $\mathrm{Ca}^{2+}, \mathrm{Mg}^{2+}$-free PBS (CMF-PBS; $156 \mathrm{mM} \mathrm{Na}^{+}, 143 \mathrm{mM} \mathrm{Cl}^{-}, 4 \mathrm{mM}$ $\mathrm{K}^{+}, 10 \mathrm{mM}$ phosphate, $\mathrm{pH}$ 7.6). EDL and soleus isolated muscles were treated with collagenase type IV (Sigma Chemical Co.) for 3 
min at room temperature. Bundles of three to four fibers were dissected under a stereomicroscope and attached onto 20-mm-diameter round glass coverslip previously treated for $30 \mathrm{~min}$ with poly-D-lysine $(1 \mathrm{mg} / \mathrm{ml})$.

Functional studies were performed on water plasma membrane permeability using the TIR microfluorometry technique (17). Chloromethyl-benzoyl-amino-tetramethyl-rhodamine (CMTMR) was used as membrane impermeant fluorophore to label the aqueous phase of the cytoplasm (18). Before microscopy measurements, the fibers were washed with CMF-PBS and incubated with $10 \mu \mathrm{M}$ of CMTMR. Fibers were initially perfused with regular CMF-PBS and then subjected to hypo/hyperosmotic treatment by reducing/increasing the $\mathrm{NaCl}$ concentration of CMF-PBS. The time course of TIR fluorescence, measured in response to osmotic gradient, was used to assess the osmotic properties of skeletal muscle fibers.

\section{Results}

Fig. 1 shows localization of AQP4 in rat skeletal muscle by immunofluorescence. AQP4 was strongly expressed at the sarcolemma membrane of some skeletal muscle fibers (Fig. $1 A$ ), whereas it was weakly expressed or absent from the surface membrane of others. No stain was clearly detected outside the sarcolemma. The corresponding control slide (Fig. $1 \mathrm{~B}$ ), performed using antibody previously reacted with the immunizing peptide, showed no labeling, confirming the specificity of the stain. Immunolocalization of AQP4 in both human (see below) and rabbit (not shown) skeletal muscles gave the same results.

To better analyze the mosaicism in the expression of AQP4, fast- and slow-twitch muscles were analyzed. For this purpose rat soleus and rat EDL were used. The large majority of rat soleus is made of slow oxidative fibers and only $15 \%$ are fast fibers. In contrast, EDL is comprised of a pure population of fast-twitch fibers. The immunofluorescence (Fig. $2 A$ ) revealed a low number of immunopositive fibers for AQP4 in soleus. In some areas of the soleus section no staining was detected. In contrast, uniform staining in the sarcolemma of all fibers both in cross-sections (Fig. $2 \mathrm{~B}$ ) and longitudinal sections (Fig. $2 C$ ) of EDL was found, demonstrating that the expression of AQP4 is continuous along the sarcolemma of fast fibers. These data show that the expression of AQP4 in skeletal muscle is fiber-type specific.

To confirm the specificity of AQP4 expression and to correlate it with muscle and fiber metabolism, combined immunofluorescence and histochemical experiments were performed on the same section of human rectum abdominal muscle. For this purpose COX enzyme activity, marker for mitochondria, was determined (Fig. 3). Muscle fibers with little or no COX activity (Fig. $3 B$ ) were fast fibers and corresponded to the ones that expressed AQP4 (Fig. $3 A$ ). In contrast, fibers with high COX activity were slow fibers and did not express AQP4 protein.

The precise cellular localization of AQP4 in EDL and soleus muscle fibers was examined by EM gold-labeling with affinity-purified anti-AQP4 antibodies (Fig. 4). In the EDL, a strong labeling was observed along plasma membranes of most fibers (Fig. 4 a, arrowheads). The density of gold labeling was variable from fiber to fiber. Fig. $4 a$ shows two adjacent fibers (top and bottom), with more plasma membrane labeling of the top fiber than the bottom fiber. No labeling was observed in intracellular structures and we found no evidence of $\mathrm{T}$ tubule labeling. In contrast to EDL, little or no labeling was observed
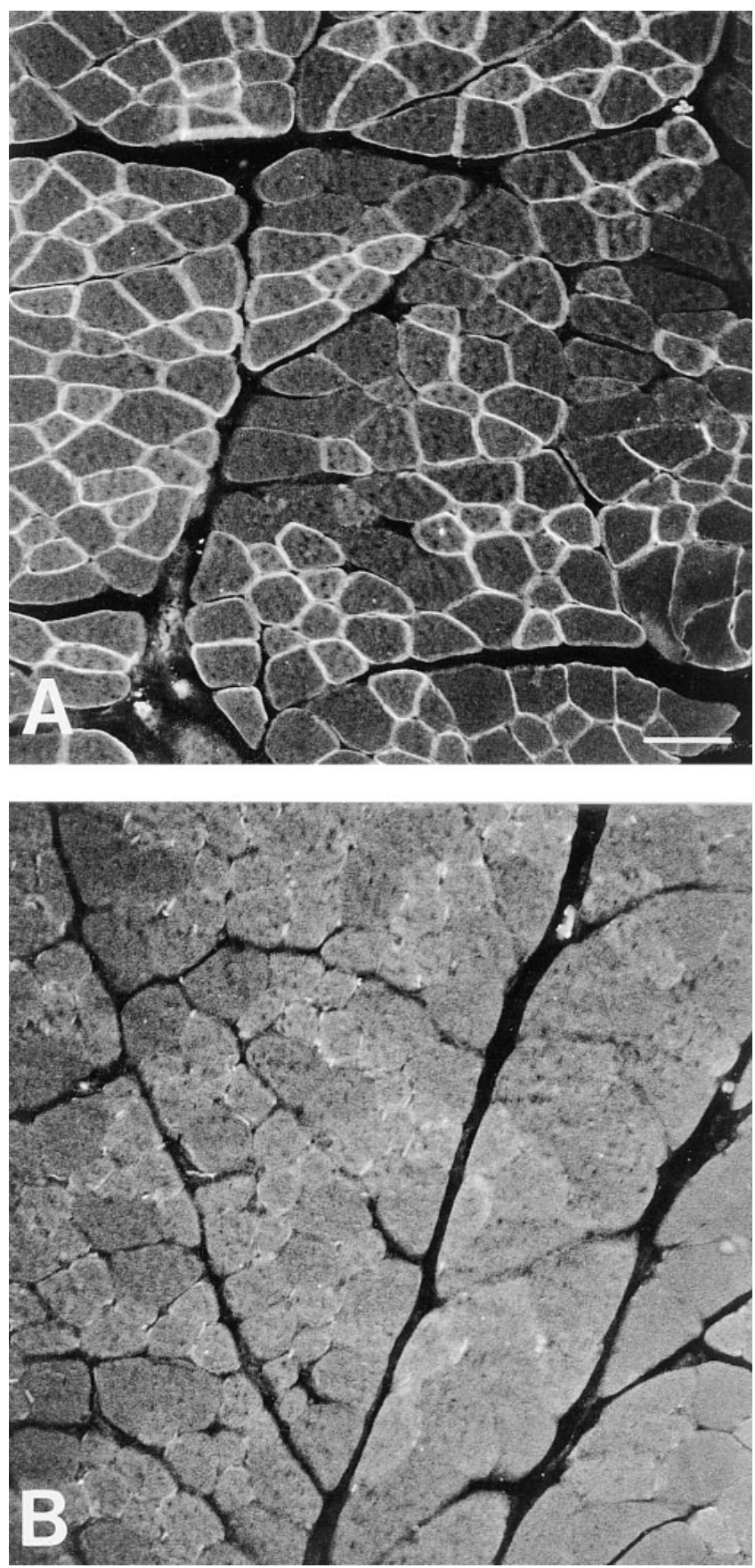

Figure 1. Immunolocalization of AQP4 in rat skeletal muscle. A cryostat section of rat intercostal muscle was stained with affinitypurified anti-AQP4 antibodies $(A)$. The labeling is localized at the sarcolemma membrane. Note the mosaicism in the expression of AQP4: some fibers are strongly fluorescent while others appear not stained. $(B)$ Immunodepleted control skeletal muscle section. Scale bar, $150 \mu \mathrm{m}$.

in muscle fiber from the soleus (Fig. $4 b$ ), either at plasma membranes (arrowheads) or in intracellular organelles.

The expression of AQP4 in skeletal muscle was also analyzed at the messenger level. RT-PCR experiments were performed using total RNA prepared from fast and slow rat muscles and from brain as control. Primers were designed to 

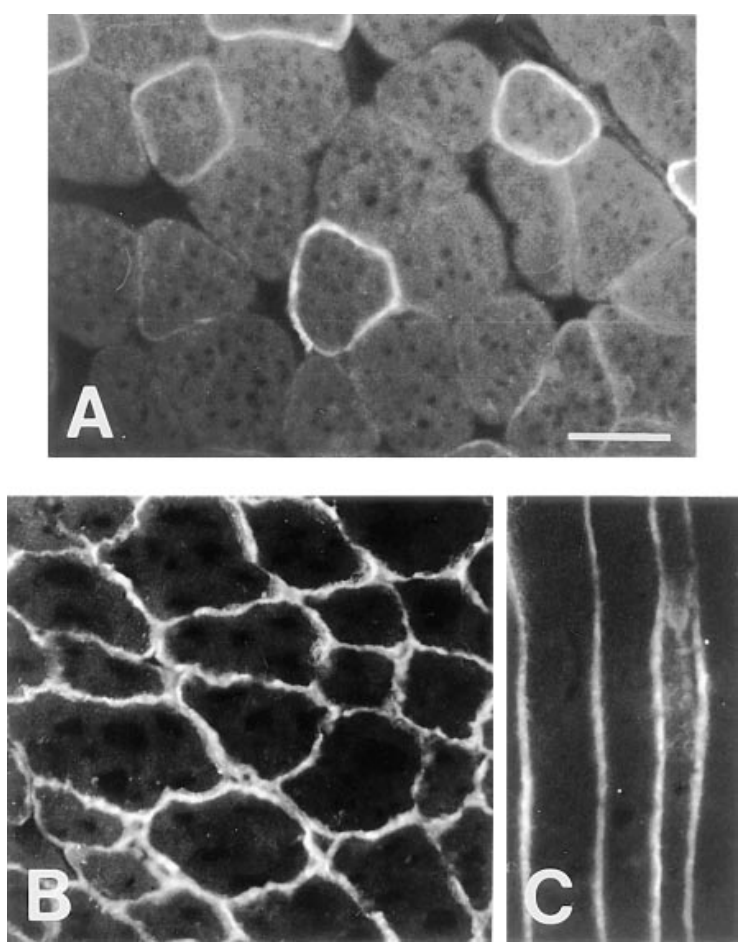

Figure 2. Characterization of AQP4 expression in fast and slow muscles. $(A)$ A transverse section of rat soleus where only few fibers were immunostained. $(B)$ A transverse section of rat EDL and $(C)$ EDL fibers cut longitudinally. Note as in EDL the staining is continuous and involves all fibers. Affinity-purified antibodies were used. Scale bar, $100 \mu \mathrm{m}$.

amplify a cDNA fragment of $960 \mathrm{bp}$ including the open reading frame of the AQP4 mRNA. The results of agarose gel electrophoresis of representative PCR reaction products are shown in Fig. $5 A$. As expected, rat brain RNA resulted in the amplification of a cDNA band of the predicted size for AQP4. A similar size band was detected in the sample containing RNA from EDL, whereas a very weak signal of AQP4 mRNA was detected in soleus. The specificity of the PCR amplification was verified using Southern blot and restriction enzyme analysis. Southern blot analysis of the PCR products shown in Fig. $5 \mathrm{~B}$ confirmed the specificity of the result and revealed, in addition, the presence in both EDL and brain of a 500-bp band scarcely visible in the ethidium bromide-stained agarose gel. Moreover, the two cDNA fragments were isolated from the agarose gel and the restriction enzyme sensitivity was determined. Experiments performed using restriction enzymes specific for each exon of the AQP4 gene indicated that the 960-bp DNA fragment contained the full-length rat AQP4 and provided no evidence that the 500-bp fragment is an authentic splice variant.

To verify that the differences found in the expression of AQP4 between EDL and soleus were not due to differences in quality and/or quantity of cDNA, we used the histone $3.3 \mathrm{a}$ as internal standard (Fig. $5 \mathrm{~A}$ ). The similar PCR amplification of histone 3.3a validated the difference found in the expression of AQP4 mRNA in EDL and soleus and confirmed the fibertype specificity of AQP4.

To characterize which form of AQP4 protein is expressed
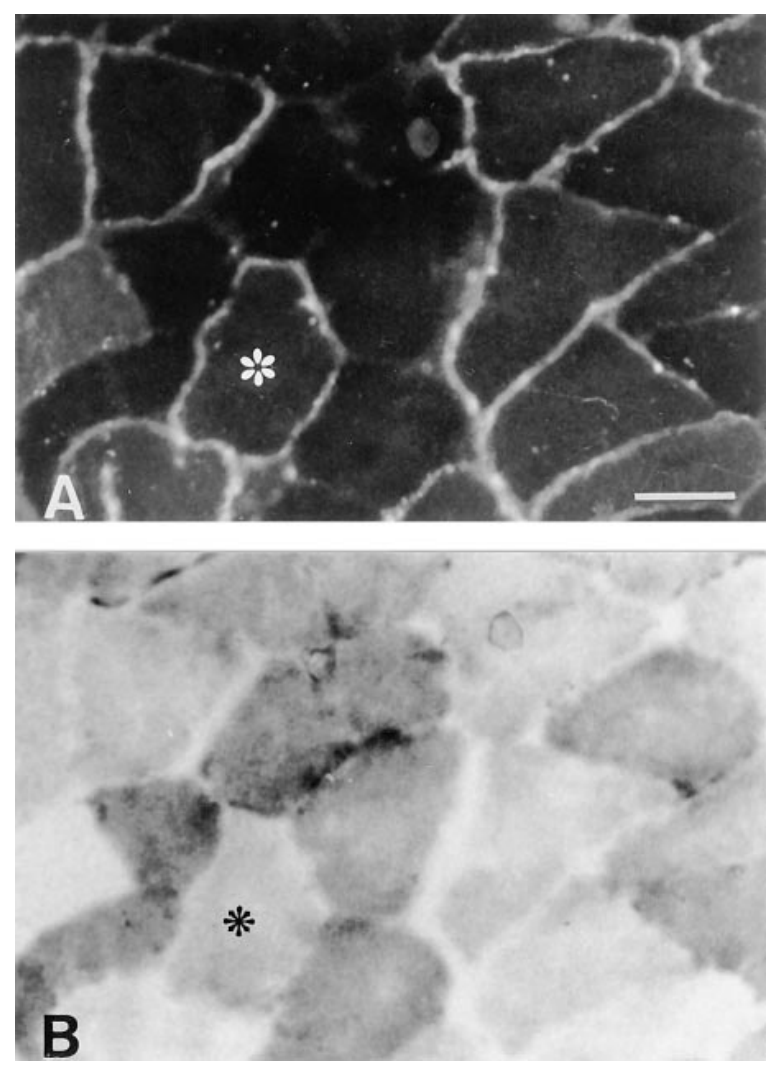

Figure 3. Characterization of fiber-type specificity of AQP4 expression in human skeletal muscle. The same section was assayed for AQP4 expression by immunofluorescence $(A)$ and for COX activity by histochemistry $(B)$. All slow fibers have strong COX activity and stain darkly but do not express AQP4. In contrast, fast fibers which have low COX activity (*) are recognized by AQP4 antibodies. Affinity-purified antibodies were used. Scale bar, $50 \mu \mathrm{m}$.

in skeletal muscle, crude homogenates from rat skeletal muscle and brain and membrane fractions were prepared by differential centrifugations at 4,000 $\mathrm{g}, 17,000 \mathrm{~g}$, and 100,000 $\mathrm{g}$. The results of Western blot analysis are shown in Fig. 6. Consistent with previous reports $(6,19)$, high levels of immunoreactive AQP4 were found in rat brain (Fig. $6 A$ ). Two prominent immunopositive bands were seen at 30 and $32 \mathrm{kD}$, confirming the presence of two splice variants. Total skeletal muscle showed similar results. The same doublet was revealed in the lane containing membranes obtained after centrifugation at 100,000 $\mathrm{g}$. Instead, only a $25-\mathrm{kD}$ band was strongly revealed in membrane preparations obtained by low speed centrifugation $(4,000 \mathrm{~g})$. To determine whether the $25-\mathrm{kD}$ protein, strongly recognized by the immune serum, was a spliced isoform of AQP4 or a cross-reacting protein, Western blot experiments were performed using fast and slow skeletal muscle membrane preparations obtained after centrifugation at 4,000 g. Fig. $6 \mathrm{~B}$ shows a representative immunoblot containing membranes from EDL and soleus muscles. Fast skeletal muscle membranes exhibited high levels of the immunoreactive $25-\mathrm{kD}$ protein, whereas low levels of the same protein were detected in soleus membranes, suggesting that this protein is selectively expressed in fast-twitch fibers. To verify whether the $25-\mathrm{kD}$ protein is related to AQP4, sequence analysis was performed. 


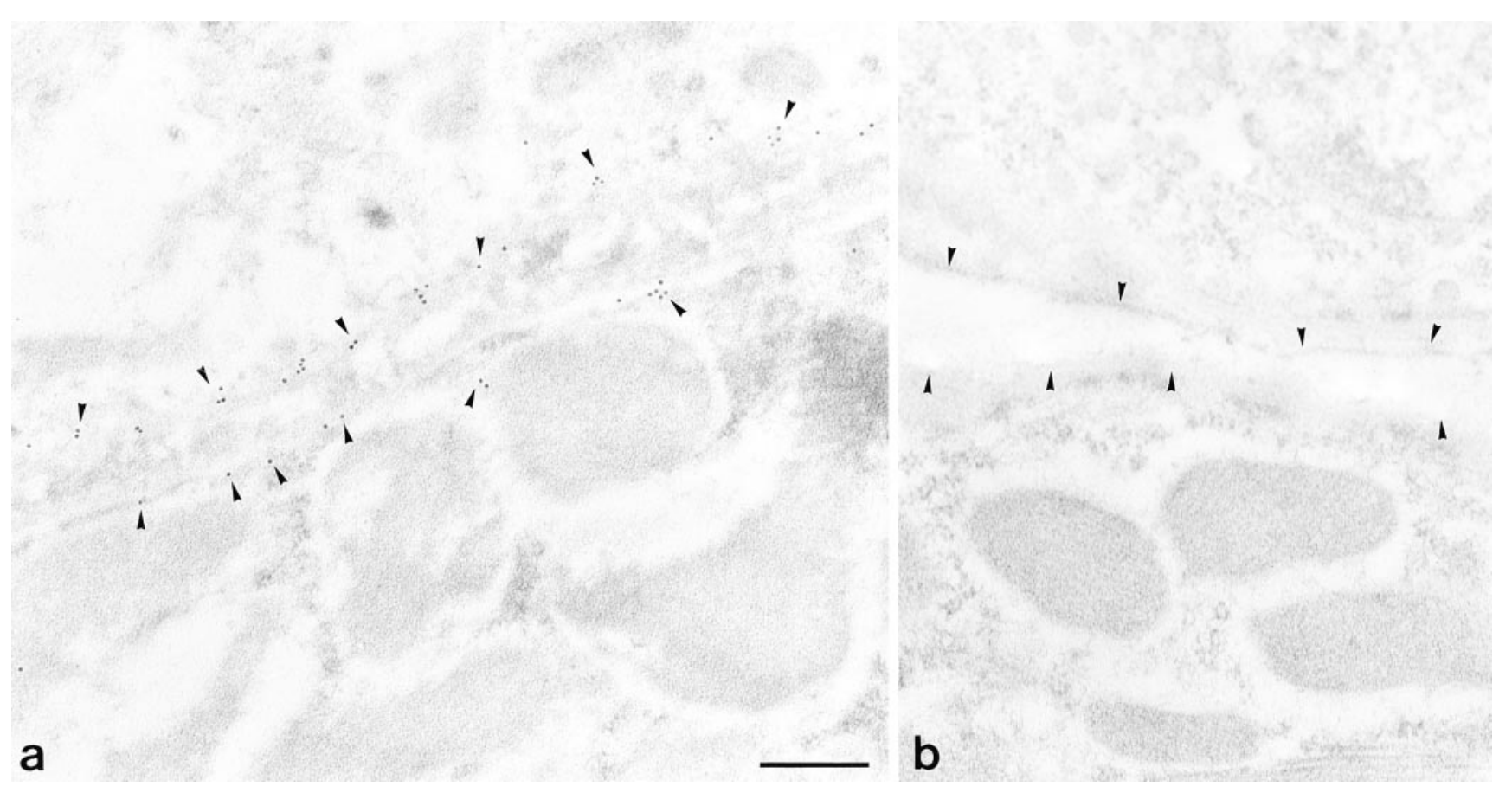

Figure 4. Localization of AQP4 in muscle cells by EM gold labeling. (a) In the EDL, labeling with affinity-purified anti-AQP4 antibodies was found along plasma membranes (arrowheads) and provided no evidence for labeling of other structures. (b) Little or no labeling was observed in muscle cells from the soleus, even along plasma membranes (arrowheads). Scale bar, $0.3 \mu \mathrm{m}$.

After in situ digestion, peptides were purified by HPLC and sequenced. The obtained partial sequence of two peptides revealed that the $25-\mathrm{kD}$ protein was the fast-twitch isoform of the Troponin I which incidentally cross-reacts with the immune serum.

Immunoblots were performed to study the fiber expression of the $30-32-\mathrm{kD}$ proteins. To this end a $100,000 \mathrm{~g}$ membrane

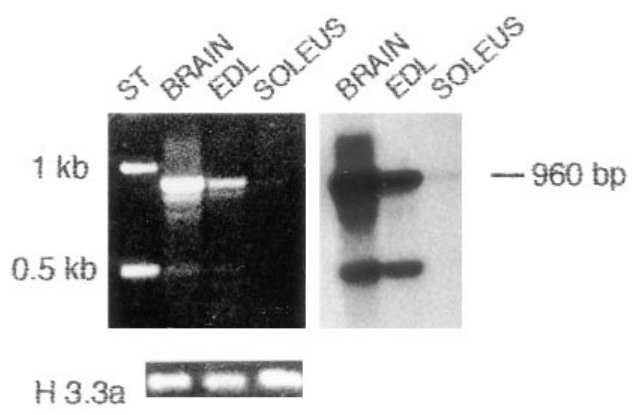

A

Figure 5. RT-PCR Southern examination of mRNA for evidence of AQP4 expression. RNA samples from rat brain, EDL, and soleus were subjected to reverse transcription followed by PCR amplification and Southern blot. The predicted PCR product $(A)$ was a fragment of $960 \mathrm{bp}$ which was amplified in brain and EDL, whereas cDNA from soleus yielded a negative result. $(B)$ Southern blot analysis of PCR products obtained probing the membrane with the fulllength AQP4 cDNA coding sequence. In addition to the 960-bp band, a smaller PCR product of $500 \mathrm{bp}$ is strongly revealed. Amplification of the histone 3.3a ( $H 3.3 a)$ was used as an internal standard. fraction from EDL, soleus, and heart was analyzed and a typical immunoblot is shown in Fig. $6 C$. The two immunopositive bands were clearly revealed in brain and EDL membranes, whereas no immunopositive bands were seen in soleus and heart preparations. Loading of all muscular lanes was equal as assessed by Coomassie blue staining of comparably loaded gels (Fig. $6 \mathrm{D}$ ). To obtain a similar revelation between brain and EDL, about 20 times more membrane proteins were needed for EDL than for brain, confirming the high expression of AQP4 in brain. In some experiments, higher molecular weight bands, representing aggregates or oligomers of AQP4, were revealed in both brain and EDL membranes. Taken all together, these data confirm the selectivity of the expression of AQP4 in fast-twitch skeletal muscle.

Based on the experiments described above, we analyzed the osmotic behavior of fast and slow muscle fibers. TIR microfluorometry was used to continuously measure volume changes of isolated muscle fibers attached to a glass coverslip and stained with CMTMR, a fluid-phase fluorophore. As previously reported (17), the recorded emitted fluorescence is inversely related to the cell volume. Fig. 7 illustrates a representative fluorescence time course of an osmotic volume change of isolated EDL fibers perfused with solutions of indicated osmolarities. The TIR fluorescence signal was dependent on perfusate osmolarity; the signal decreased upon perfusion with hypotonic solution as a consequence of water influx and cell swelling. Instead, the fluorescence signal increased with a hypertonic solution due to cell shrinkage. After the bathing solution was switched, cell volume smoothly approached a new steady state and the swelling or shrinkage was fully reversible.

To compare the osmotic properties of fast- and slow-twitch muscles, parallel TIR experiments were performed using EDL 


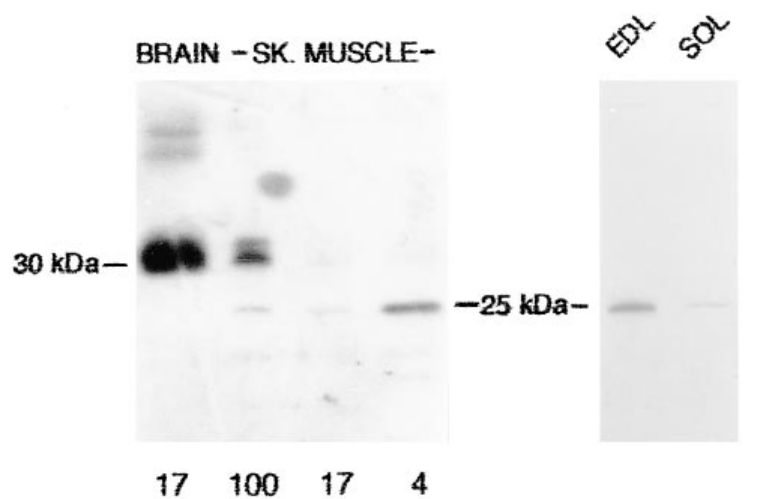

A

B

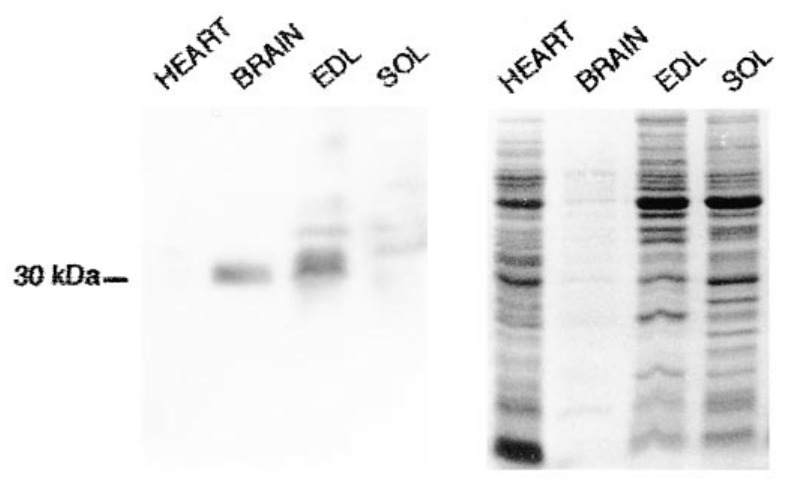

C

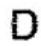

Figure 6. Immunoblot analysis of AQP4 protein expression. Membrane proteins were separated by SDS-PAGE on a $13 \%$ acrylamide gel, transferred to a PVDF membrane, and probed with AQP4 immune serum. $(A)$ Membrane fraction from brain $(10 \mu \mathrm{g})$ and skeletal muscle (60 $\mu \mathrm{g})$ using 4,000 $g(4), 17,000 g(17)$, and 100,000 $g(100)$ membrane pellets showing the same two immunopositive bands at a $30-\mathrm{kD}$ region in brain and in 100,000 $\mathrm{g}$ muscle membranes, whereas a $25-\mathrm{kD}$ protein band is recognized in the $4,000 \mathrm{~g}$ pellet. $(B)$ Immunoblot of membrane fractions from rat EDL and soleus using 4,000 $\mathrm{g}$ pellets showing a strong expression of the $25-\mathrm{kD}$ band in EDL membranes. (C) Immunoblot of membrane proteins using 100,000 $g$ pellets membrane proteins showing that the two immunopositive bands are present in brain and EDL and not in heart and soleus. (D) Coomassie blue-stained gel. Less total brain protein was electrophoresed.

and soleus fibers from the same rat. The kinetics of osmotic volume changes were characterized by comparing the time constants, for swelling or shrinkage, obtained from the experimental data fitted with a single exponential function. Isolated EDL and soleus fibers were subjected to an osmotic swelling. Representative superimposed curves are shown in Fig. $8 \mathrm{~A}$ in which the curve for EDL fibers shows a faster osmotic response compared with that for soleus fibers. The results are summarized in the histogram shown in Fig. 8 B. The time constant obtained from soleus was approximately sixfold larger than that obtained from EDL fibers.

Temperature dependence studies were carried out to get

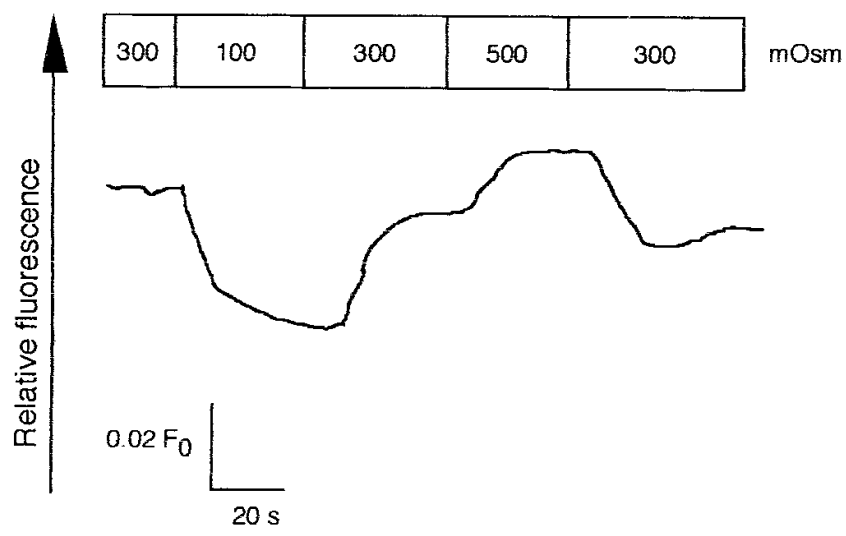

Figure 7. Time course of swelling and shrinkage of EDL fibers in response to changes in bath osmolality. Fibers were loaded with CMTMR fluorescent dye and then perfused at $10 \mathrm{ml} / \mathrm{min}$ perfusion rate with $\mathrm{PBS}$ at $20^{\circ} \mathrm{C}$. Bath osmolality is indicated.

an insight into the mechanism of water permeation in skeletal muscle. The effect of temperature on the osmotic volume changes in soleus and EDL fibers is summarized in Fig. 9. A nonsignificant temperature dependence was observed for the time constant of the osmotic swelling of EDL fibers, suggesting a water channel-mediated transport. In contrast, the time

A

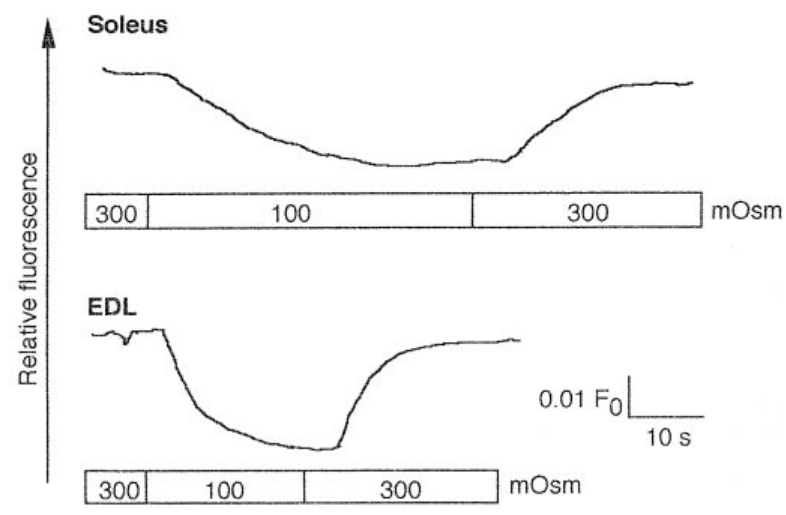

$\mathrm{B}$

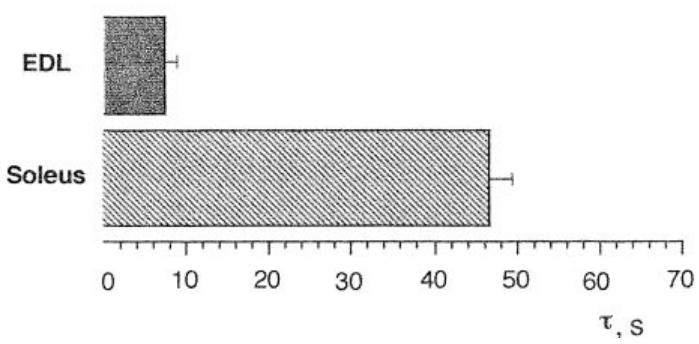

Figure 8. Osmotic properties of soleus and EDL fibers measured by TIR fluorescence. $(A)$ Representative TIR fluorescence time course in response to a 200-mosmol inwardly directed $\mathrm{NaCl}$ gradient at $20^{\circ} \mathrm{C}$. (B) Mean values \pm SE of the time constant $(n=15)$. 


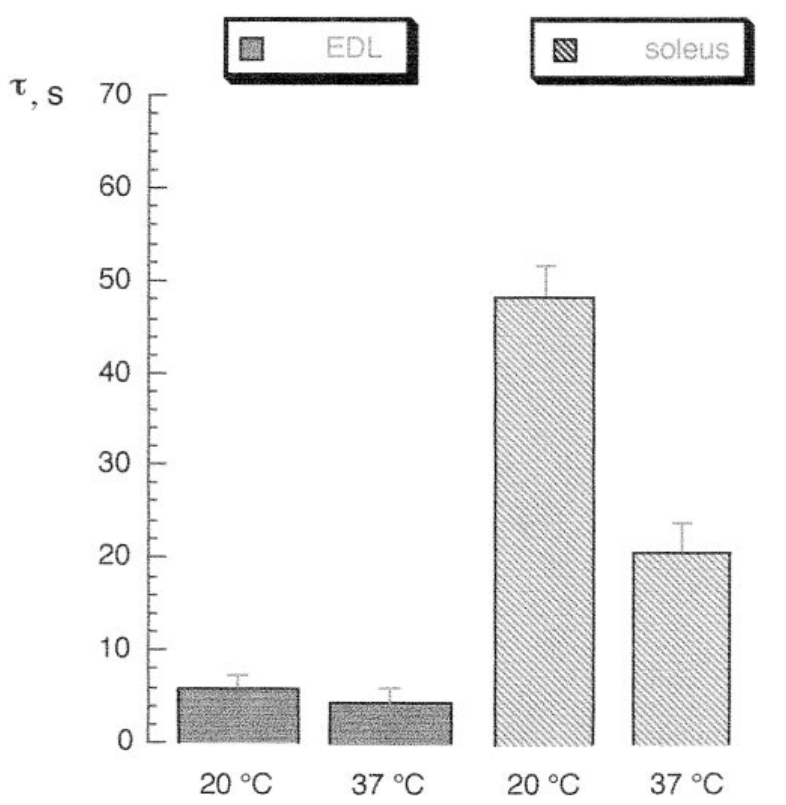

Figure 9. Temperature-dependent changes of EDL and soleus fiber volume. The effect of temperature was determined at 20 and $37^{\circ} \mathrm{C}$ using $\tau$ of fibers swelling (mean values \pm SE, $n=15$ ). Osmotic swelling of soleus fibers was more affected by temperature.

constant of soleus fibers was more temperature dependent, indicating that osmotic property differences exist between the two skeletal muscle fibers.

Another approach for examining this issue is the determination of the coefficient $\mathrm{Q}_{10}$. This coefficient indicates the increase in the transport rate occurring when the temperature is elevated $10^{\circ} \mathrm{C}$. For the diffusion mechanism of water transport through a lipid bilayer, the $\mathrm{Q}_{10}$ for $\mathrm{Pf}$ is $\sim 2$ (20). In contrast, for water flow through an aqueous pore, the $\mathrm{Q}_{10}$ will essentially be that for the viscosity of water, which is $\sim 1.25$. The $\mathrm{Q}_{10}$ obtained from the two different skeletal muscle fibers and calculated using the time constant was $1.2 \pm 0.1$ for EDL and $1.8 \pm 0.2$ for soleus fibers.

Previous freeze-fracture studies of skeletal muscle from patients with DMD and from mdx mice showed alteration in the internal molecular architecture of the muscle plasma membrane and in particular a marked depletion of OAPs has been observed (11). Since AQP4 has been proposed to be a component of OAPs $(6,21)$, we analyzed the expression of AQP4 in the skeletal muscle of mdx mouse, the animal model of DMD. Fig. 10 shows immunofluorescence analysis of a fast-twitch skeletal muscle from an $\mathrm{mdx}$ mouse. A marked reduction of the AQP4 staining was observed in the mdx mouse (Fig. 10 B) compared with the age-matched control (Fig. $10 A$ ). As expected, the dystrophin immunostaining was found to be normal in the control mouse (Fig. $10 C$ ) and absent in the $\mathrm{mdx}$ mouse (Fig. $10 \mathrm{D}$ ). Moreover, immunofluorescence experiments performed to check the cytoskeleton and plasma membrane integrity (not shown) confirmed that the reduction of the AQP4 staining in mdx mouse was not due to nonspecific secondary effects of muscle fiber degeneration. These results suggest the possible involvement or role of AQP4 in the membrane alteration of DMD.

\section{Discussion}

Recent immunolocalization studies have demonstrated the expression of AQP4 in several tissues where water transport is important including kidney, lung, eye, and stomach $(5,6,22)$. However the predominant site of expression of AQP4 is the neuromuscular system. The high expression of AQP4 in astrocytes throughout the central nervous system and in ependymal cells lining the brain ventricles and pia suggested a role of AQP4 in the regulation of water flux and volume control in brain (23). Expression of AQP4 has also been reported in skeletal muscle $(6,24)$. The physiological role of AQP4 in skeletal muscle can only be understood after definition of the precise cellular distribution. Therefore, the purpose of this study was to analyze in detail the expression of AQP4 and to verify, by functional analysis, whether the expression of AQP4 can contribute to the osmotic water flow in skeletal muscle.

A key finding in this study was the strong expression of AQP4 at the sarcolemma membrane of fast-twitch fibers. All experiments performed by us consistently supported this conclusion (i.e., immunomicroscopy, RT-PCR, and Western blot analysis). This conclusion is further validated by the isolation from a human skeletal muscle cDNA library of two clones containing the full sequence for AQP4 (our unpublished data).

The presence of water channels in skeletal muscle has been the subject of much debate. In the old literature, an increase of water penetration was found in ADH-stimulated frog muscle fibers (25). Sorenson (26) reported high water permeability for skeletal muscle fibers isolated from a marine crab although the data probably were influenced by an underestimation of sur-
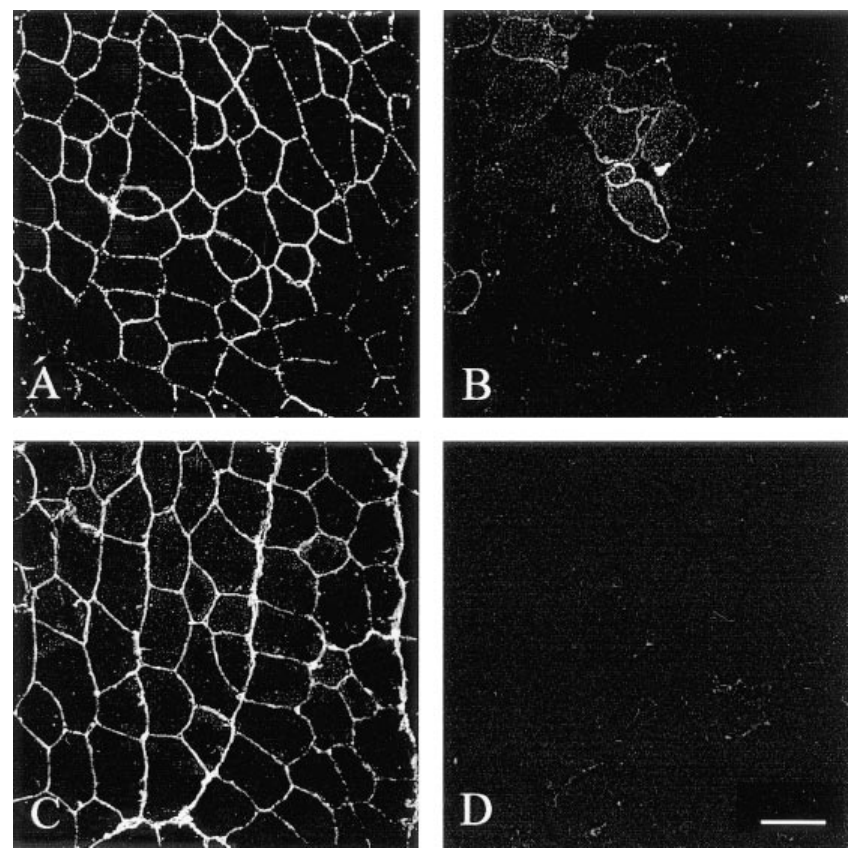

Figure 10. Immunofluorescence analysis of AQP4 expression in skeletal muscle of mdx mouse. Tibialis anterior muscles from control $(A)$ and mdx mice $(B)$ were stained with AQP4 affinity-purified antibodies. Dystrophin immunostaining (Sigma Chemical Co.; 1:100) is shown in control $(C)$ and mdx mice $(D)$. Scale bar, $100 \mu \mathrm{m}$. Note the reduced number of fibers stained by AQP4 antibodies in the mdx mouse $(B)$. 
face area. More recently, Berman (27) reported low water permeability in giant barnacle skeletal muscle, but complex invagination of the barnacle sarcolemma and the effect of unstirred layers, favored by the large diameter of barnacle muscle cells, probably lead to an incorrect estimation of water permeability coefficient. The most detailed analysis was made recently by Suleymanian and Baumgarten (28) to assess water permeation in cardiac muscle. Functional studies performed in cardiac myocytes revealed that water channels do not significantly contribute to sarcolemma water permeability and that the primary route for water crossing cardiac membranes is likely to be directly through the lipid bilayer itself. In line with this report our results show no expression of AQP4 in heart.

We now present evidence that in skeletal muscle AQP4 is strongly expressed in fast contracting fibers. This conclusion comes from experiments performed using rat EDL and soleus since these two muscles have different fiber composition (29). EDL is composed of fast-twitch glycolytic (FG) and fast-twitch oxidative glycolytic fibers (FOG) in a similar number. Immunofluorescence performed in EDL revealed that all fibers were labeled by AQP4 antibodies, indicating that both FG and FOG fibers expressed AQP4 protein. In contrast, soleus is made of $15 \%$ of FOG fibers and $85 \%$ of slow-twitch oxidative (SO) fibers. In this case, immunofluorescence revealed only a few fibers expressing AQP4, likely the FOG. Combined histochemical and immunofluorescence analysis performed on human skeletal muscle confirmed that SO fibers did not express AQP4. In fact, since FOG fibers are rare in the human, COX positives are only SO fibers and are not labeled by AQP4 antibodies. EM gold labeling in muscular tissue confirmed that AQP4 is abundant in fibers from EDL although the density of gold labeling varied from fiber to fiber, suggesting a cellular heterogeneity of the expression of AQP4 in this tissue. Although AQP4 seems to be not expressed in slowtwitch fibers, we cannot rule out the possibility that these fibers express another still unknown water channel. As shown in results, the quantity of AQP4 protein expressed in skeletal muscle seems to be much lower (20 times) than in brain. Further studies are required to determine the physiological role of AQP4 in skeletal muscle in terms of its contribution to membrane water permeability as well as comparison studies in mammals in which the protein is nonfunctional because of natural mutation or in transgenic knockouts. Interestingly it has been reported that stimulators of protein kinase $\mathrm{C}$ and protein kinase A, respectively, decreases (30) the AQP4 activity or increases (31) the appearance of OAPs, the morphological equivalent of AQP4, suggesting that a potential regulation of AQP4 can occur in vivo.

The highly selective location of AQP4 at the sarcolemma membrane of fast-twitch fibers would imply that the physiological role of AQP4 in skeletal muscle may be linked to fiber metabolism.

Many factors have been proposed to explain the mechanisms behind fatigue and exhaustion in muscular exercise. Osmolality changes and water fluxes during muscle activity have been reported extensively. At the onset of an intense muscle exercise, intracellular lactate concentration increases and may reach $41 \mathrm{mM}$ in a few seconds, becoming the greatest single contributor to the osmotic changes (12). A high correlation has been observed between the increase in muscle lactate and water content $(14,32)$. The water displacement as result of lactate accumulation causes secondary changes in the concentrations and gradients of ions and other metabolites. Since lactate accumulation in rat and human glycolytic fibers is higher than in oxidative fibers, the presence of a water channel at the sarcolemma membrane of fast-twitch fibers may be of importance in muscle volume regulation. AQP4 could determine a rapid and efficient transfer of water from the interstitial space into the fiber allowing an immediate volume adaptation after an intense muscle exercise. If so, the functional significance of this rapid adaptation needs to be determined.

It has been proposed that limitation occurring in muscle fatigue should be sought in the excitation-contraction coupling. One possibility could be that during exercise the alterations in water and electrolyte concentrations, such as $\mathrm{K}^{+}$concentration across the membranes, are of such a magnitude as to affect the membrane potential and thus the magnitude of the action potential and the propagation velocity. In fact, during contraction $\mathrm{K}^{+}$is continuously released from the muscle $(14,32)$. The nature of the $\mathrm{K}^{+}$loss from the muscle during exercise has been attributed to an incomplete reuptake of $\mathrm{K}^{+}$with each depolarization (33). Thus, muscle fatigue may then be related to changes in $\mathrm{K}^{+}$gradient and water concentration across the sarcolemma membrane and not only to energy metabolism.

Potassium release is not unique to skeletal muscle. Recent studies show that primary cultures of astrocytes manifest an increase of $\mathrm{K}^{+}$conductance during hypoosmotic swelling (34). This may mediate diffusive loss of $\mathrm{K}^{+}$from astrocytes during volume regulation after hypoosmotic swelling. The fact that AQP4 is also expressed in astrocytes suggests a special function in these two cell types; AQP4 would allow a quick response to changes in cellular osmolarity.

The physiological consequences of the presence of a water channel in skeletal muscle remain unknown. We can speculate that in muscle the presence of AQP4 would be important to establish muscle fatigue and to protect the cell against overload. Since it is likely that some muscle fibers are more heavily engaged during fatiguing work than others, it is possible that the most active fibers (FG and FOG) would be affected to a much larger extent and AQP4 in these fibers may have a role in such a process.

The sites where AQP4 has been localized correspond to membranes were orthogonal arrays of particles have been observed by freeze-fracture electron microscopy (7). Present data further validate the hypothesis that AQP4 is the OAP protein. As recently proposed (35), the assembly of AQP4 in orthogonal arrays could have the effect of enhancing water flow, which could mediate rapid changes in cell volume in response to local shifts in osmolality.

Modification of the OAP membrane density has been reported in various pathological states such as epilepsy, brain ischemia, and muscular dystrophy.

In previous freeze-fracture studies, undertaken to investigate the changes in muscle plasma membrane architecture of skeletal muscle, a decrease in OAPs density in DMD plasma membrane has been described previously (11). A similar change is noted in other human muscular dystrophies, such as the Fukuyama (36) and the facioscapulohumeral type (37), and in animal models of muscular dystrophy, e.g., mdx mouse (38). This suggested that a common denominator of dystrophic myofiber plasma membrane change is the depletion of orthogonal arrays. Therefore, we analyzed the expression of AQP4 in the skeletal muscle of the mdx mouse. Experiments performed in mdx mice have shown a drastic reduction of the AQP4 
immunostaining, suggesting that AQP4 could be involved in the pathophysiology of the muscle disease. Further experiments are needed to verify whether the decreased AQP4 expression takes place at the messenger level or at the protein level and whether this is a causal factor contributing to the pathology of the muscle disease or a secondary phenomenon manifested by a diseased muscle.

As shown for other tissues, insight into the properties, regulation, and localization of AQP4 in skeletal muscle may provide a key to understanding how volume homeostasis is normally achieved and how it might be restored if pathologically perturbed.

\section{Acknowledgments}

We thank Dr. M. Capozzi (Clinica S. Maria, Bari, Italy) for supplying the human skeletal muscle biopsies, Dr. R. Filippetti (Policlinico Gemelli, Rome, Italy) for providing the mdx mice and Prof. O. Hutter (Glasgow University, Glasgow, United Kingdom) for discussion.

The financial support of Telethon-Italy (grant no. 983) is gratefully acknowledged.

\section{References}

1. Verkman, A.S., A.N. Van hoek, T. Ma, A. Frigeri, W.R. Skach, A. Mitra, B.K. Tamarappoo, and J. Farinas. 1996. Water transport across mammalian cell membranes. Am. J. Physiol. 39:C12-C30.

2. King, L.S., and P. Agre. 1996. Pathophysiology of the aquaporin water channels. Annu. Rev. Physiol. 58:619-648.

3. Hasegawa, H., T. Ma, W. Skach, M.A. Matthay, and A.S. Verkman. 1994. Molecular cloning of a mercurial-insensitive water channel expressed in selected water-transporting tissues. J. Biol. Chem. 269:5497-5500.

4. Jung, J.S., R.V. Bhat, G.M. Preston, W.B. Guggino, J.M. Baraban, and P. Agre. 1994. Molecular characterization of an aquaporin cDNA from brain: candidate osmoreceptor and regulator of water balance. Proc. Natl. Acad. Sci. USA. 91:13052-13060.

5. Frigeri, A., M.A. Gropper, C.W. Turck, and A.S. Verkman. 1995. Immunolocalization of the mercurial-insensitive water channel and glycerol intrinsic protein in epithelial cell plasma membranes. Proc. Natl. Acad. Sci. USA. 92: 4328-4331.

6. Frigeri, A., M.A. Gropper, F. Umenishi, M. Kawashima, D. Brown, and A.S. Verkman. 1995. Localization of MIWC and GLIP water channel homologues in neuromuscular, epithelial and glandular tissues. J. Cell Sci. 108:29933002.

7. Yang, B., D. Brown, and A.S. Verkman. 1996. The mercurial insensitive water channel (AQP-4) forms orthogonal arrays in stably transfected Chinese hamster ovary cells. J. Biol. Chem. 271:4577-4580.

8. Neuhaus, J., E.M. Schmid, and H. Wolburg. 1990. Stability of orthogonal arrays of particles in murine skeletal muscle and astrocytes after circulatory arrest, and human gliomas. Neurosci. Lett. 109:163-168.

9. Suzuki, M., Y. Iwasaki, T. Yamamoto, H. Konno, T. Yoshimoto, and J. Suzuki. 1984. Disintegration of orthogonal arrays in perivascular astrocytic processes as an early event in acute global ischemia. Brain Res. 300:141-145.

10. Hatton, J.D., and M.H. Ellisman. 1984. Orthogonal arrays are redistributed in the membranes of astroglia from alumina-induced epileptic foci. Epilepsia. 25:145-151.

11. Wakayama, Y., T. Jimi, N. Misugi, T. Kumagai, S. Miyake, S. Shibuya, and T. Miike. 1989. Dystrophin immunostaining and freeze-fracture studies of muscles of patients with early stage amyotrophic lateral sclerosis and Duchenne muscular dystrophy. J. Neurol. Sci. 91:191-205.

12. Kowalchuk, J.M., G.J.F. Heigenhauser, M.I. Lidinger, J.R. Sutton, and N.L. Jones. 1988. Factors influencing hydrogen ion concentration in muscle after intense exercise. J. Appl. Physiol. 65:2080-2089.

13. van Beaumont, W., J.C. Strand, J.S. Petrofsky, S.G. Hipskind, and J.E. Greenleaf. 1973. Changes in total plasma content of electrolytes and proteins with maximal exercise. J. Appl. Physiol. 34:102-106.

14. Lindinger, M.I., L.L. Spriet, E. Hultman, T. Putman, R.S. McKelvie, L.C. Lands, N.L. Jones, and G.J. Heigenhauser. 1994. Plasma volume and ion regulation during exercise after low- and high-carbohydrate diets. Am. J. Phys- iol. 266:R1896-R1906

15. Markwell, M.A., S.M. Haas, L.L. Bieber, and N.E. Tolbert. 1978. A modification of the Lowry procedure to simplify protein determination in membrane and lipoprotein samples. Anal. Biochem. 87:206-210.

16. Seligman, A.M., M.J. Karnovsky, H.L. Wasserkrug, and J.S. Hanker. 1968. Nondroplet ultrastructural demonstration of cytochrome oxidase activity with a polymerizing osmiophilic reagent, diaminobenzidine (DAB). J. Cell Biol. 38:1-14.

17. Farinas, J., V. Simanek, and A.S. Verkman. 1995. Cell volume measured by total internal reflection microfluorometry: application to water and solute transport in cells transfected with water channel homologues. Biophys. J. 68: $1613-1620$.

18. Valenti, G., A. Frigeri, P.M. Ronco, C. D'Ettorre, and M. Svelto. 1996. Expression and functional analysis of water channels in a stably AQP2-transfected human collecting duct cell line. J. Biol. Chem. 271:24365-24370.

19. Nielsen, S., E. Arnulf Nagelhus, M. Amiry-Moghaddam, C. Bourque, P. Agre, and O. Petter Ottersen. 1997. Specialized membrane domains for water transport in glial cells: high-resolution immunogold cytochemistry of aquaporin-4 in rat brain. $J$. Neurosci. 17:171-180.

20. Finkelstein, A. 1985. Water Movement Through Lipid Bilayers, Pores, and Plasma Membranes. Theory and Reality. Wiley Interscience, New York. $228 \mathrm{pp}$

21. Verbavatz, J.M., T. Ma, R. Gobin, and A.S. Verkman. 1997. Absence of orthogonal arrays in kidney, brain and muscle from transgenic knockout mice lacking water channel aquaporin-4. J. Cell Sci. 110:2855-2860.

22. Folkesson, H.G., M.A. Matthay, A. Frigeri, and A.S. Verkman. 1996. Transepithelial water permeability in microperfused distal airways. Evidence for water channel-mediated water transport. J. Clin. Invest. 97:664-671.

23. Lu, M., M.D. Lee, B.L. Smith, J.S. Jung, P. Agre, M.A. Verdijk, G. Merkx, J.P. Rijss, and P.M. Deen. 1996. The human AQP4 gene: definition of the locus encoding two water channel polypeptides in brain. Proc. Natl. Acad. Sci. USA. 93:10908-10912.

24. Yang, B., T. Ma, and A.S. Verkman. 1995. cDNA cloning, gene organization, and chromosomal localization of a human mercurial insensitive wate channel. Evidence for distinct transcriptional units. J. Biol. Chem. 270:2290722913.

25. Zunaiski, J.A., M.N. Parisi, and R. Montoreano. 1963. Effect of antidiuretic hormone on permeability of single muscle fibers. Nature. 200:365-366.

26. Sorenson, A.L. 1971. Water permeability of isolated muscle fibers of a marine crab. J. Gen. Physiol. 58:287-303.

27. Berman, D.M., C. Pena-Rasgado, M. Holmegren, P. Hawkins, and H. Rasgado-Flores. 1993. External Ca effect on water permeability, regulatory volume decrease, and extracellular space in barnacle muscle cells. Am. J. Physiol. 265:C1128-C1137.

28. Suleymanian, M.A., and C.M. Baumgarten. 1996. Osmotic gradientinduced water permeation across the sarcolemma of rabbit ventricular myocytes. J. Gen. Physiol. 10:503-514.

29. Armstrong, R.B., and R.O. Phelps. 1984. Muscle fiber type composition of the rat hindlimb. Am. J. Anat. 171: 259-272.

30. Han, Z., M.B. Wax, and R.V. Patil. 1998. Regulation of aquaporin-4 water channels by phorbol ester-dependent protein phosphorylation. J. Biol. Chem. 273:6001-6004.

31. Tao-Cheng, J.H., J.P. Bressler, and M.W. Brightman. 1992. Astroglial membrane structure is affected by agents that raise cyclic AMP and by phosphatidylcholine phospholipase C. J. Neurocytol. 21:458-467.

32. Sjogaard, G., R.P. Adams, and B. Saltin. 1985. Water and ion shifts in skeletal muscle of humans with intense dynamic knee extension. Am. J. Physiol. 248:R190-R196.

33. Hirche, H., E. Schumacher, and H. Hagemann. 1980. Extracellular K ${ }^{+}$ concentration and $\mathrm{K}^{+}$balance of the gastrocnemius muscle of the dog during exercise. Pfluger's Arch. 387:231-237.

34. Olson, J.E., and G. Li. 1997. Increased $\mathrm{K}^{+}$, chloride, and taurine conductances in astrocytes during hypoosmotic swelling. Glia. 20:254-261.

35. Yang, B., A.N. van Hoek, and A.S. Verkman. 1997. Very high single channel water permeability of aquaporin-4 in baculovirus-infected insect cells and liposomes reconstituted with purified aquaporin-4. Biochemistry. 36:76257632 .

36. Wakayama, Y., T. Kumagai, and S. Shibuya. 1985. Freeze-fracture studies of muscle plasma membrane in Fukuyama-type congenital muscular dystrophy. Neurology. 35:1587-1593.

37. Schotland, D.L., E. Bonilla, and Y. Wakayama. 1981. Freeze-fracture studies of muscle plasma membranes in human muscular dystrophy. Acta Neuropathol. 54:189-197.

38. Shibuya, S. and Y. Wakayama. 1991. Changes in muscle plasma membranes in young mice with $\mathrm{X}$ chromosome-linked muscular dystrophy: a freezefracture study. Neuropathol. Appl. Neurobiol. 17:335-344. 